\title{
Resource Optimization of Mobile Intelligent System With Heart MPLS Network
}

\author{
$\underline{\text { doi:10.3991/ijim.v3s2.965 }}$ \\ Jawad OUBAHA ${ }^{1}$, Ahmed HABBANI ${ }^{1,2}$ and Mohammed Elkoutbi ${ }^{1}$ \\ ${ }^{1}$ Mohammed V-Souissi University, Rabat, Morocco \\ ${ }^{2}$ Ecole Mohammadia d'Ingénieurs, Agdal Rabat, Morocco
}

\begin{abstract}
In this paper, we introduce the original Mobile Intelligent System (MIS) in embedded FPGA architecture. This node will allow the construction of autonomous mobile network units which can move in unknowns, inaccessible or hostile environment for human beings, in order to collect data by various sensors and transmits them by routing to a unit of distant process.
\end{abstract}

For the sake of improving the performance of transmission, we propose a global schema of QoS management using DiffServ/MPLS backbones. We provide an evaluation of several scenarios for combining QoS IP networks with MIS access network. We conclude with a study on interoperability between QoS patterns in access and backbone networks.

Index Terms - Intelligent sensors, wireless sensor network, Quality of Service, DiffServ and MPLS.

\section{INTRODUCTION}

Sensors become necessary elements in all systems where information resulting from the external environment is necessary to evaluate and act. To have an exact and complete knowledge on the subject requires the deployment of several sensors, and possibly, to combine all retrieved information to have a better adjustment of each parameter's sensor.

A sensor network is composed of a large number of units called nodes. Each node is composed principally of one or several sensors, a processing unit and a module of communication, etc... These nodes communicate between each other according to the network topology and the existence or not of an infrastructure (access points) to forward the information to a control unit outside the zone of measure. All these features enable us to imagine an adaptive complex system built around several sensors in a wireless communication system. An original system has been designed and realized named MIS (Mobile Intelligent System) project, which allows integrating three main functions: acquisition, processing and routing of information around embedded architecture like FPGA.

The introduction of QoS (Quality of Service) in our project is even more complex and more challenging because their topology and resources are dynamically evolving. The need of QoS mechanisms in these environments is crucial because of limited resources, the unpredictability of the bandwidth and the high error rate. ([1] [2]).

To provide flexible services with a guarantee of performance parameters as: delay, jitter, bandwidth rates and packet loss, the IP networks must necessarily incorporate a concept of prioritization and control admission in the network. These QoS concepts must be available in all areas and access crossed networks. A backbone network with QoS can be built on a DiffServ (Differentiated Services) architecture, which offers a differentiation and prioritization based on packets classification at the entrance of the network [1]. DiffServ architecture imposes a proper design and dimensioning of the network and an optimal configuration of the involved parameters to guarantee the QoS. End-to-end QoS management implies the presence of specific mechanisms for managing QoS at different levels (network backbone and access networks). In several cases, the bottleneck is at the level of access networks. The introduction of QoS in these access networks has become more than a necessity, typically in a mobile environment. It must be present in the ingoing and outgoing edges.

In this paper, we present the architecture of MIS and the study of some problems associated with QoS in MIS interconnected with wired QoS IP networks using Diffserv or/and MPLS.

The paper is organized as follows: The first section is reserved for a general introduction. The second section focuses on the functional architecture and the experimental MIS platform and its units;

Section 3, presents the concept of quality of service, we present then the pillars of QoS in IP networks: DiffServ and MPLS.

In Section 4, simulation and an evaluation of performances is given of some scenarios of interconnection between MIS, MPLS and DiffServ. The paper ends with a conclusion and some perspectives.

\section{MIS PLATFORM}

In this section, we will present the MIS project and its experimental platform system previously introduced in [37].

\section{A. MIS presentation}

MIS (Mobile Intelligent System) is a platform of prototyping of intelligent wireless sensors elaborated within Wireless Sensor Networks (WSN) group of the Laboratory Electronic and Communication (LEC) for topological applications of networks of communicating objects. This platform is based with various sensors ( $\mathrm{CO}$, resistive tape recorder...), of a routing and treatment unit, a module of wireless radio communication using standard BLUETOOTH or WIFI and a routing and treatment unit based on a microprocessor (IP software).

\section{B. MIS applications}

One of the main applications is the construction of mobile autonomous units' network capable of moving in unknown environment, inaccessible, hostile for human 
being or in risk areas (fire, radiation, earthquake...) in order to optimize the human assistance. The aim is to provide ground information to establish a strategy of evolution according to the wished purpose. For example, we can locate victims during the rescue operations. This is possible thanks to small mobiles capable of infiltrating through rubble or others explore the watery funds, another application, and not the slightest, is the military exploitation. In this context, the use of sensors' networks allows the surveillance of the perimeters, to assist air or ground attacks and to lead operations of espionage. For that purpose, no element has to be indispensable for the functioning of the network. Such an Ad hoc architecture can maintain the network in activity after the loss of one or several elements and requires a routing module.

\section{MIS Architecture}

The functional architecture and the experimental platform MIS, is built upon the kit of development ALTERA Cyclone (System One Programmable Chip), it is composed essentially by 4 units (figure 1) : an acquisition unit, a treatment unit, a routing unit and a radio interface unit.

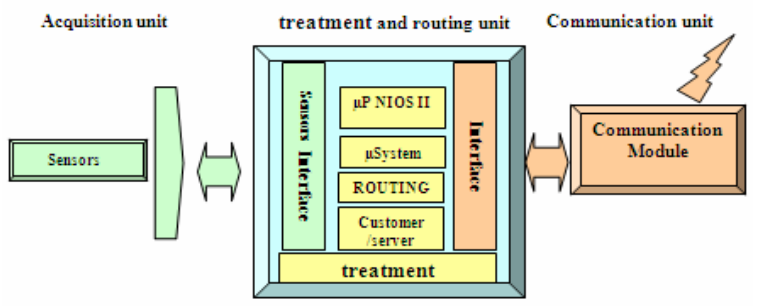

Figure 1. MIS architecture

The detailed architecture of the designed and produced beacon is given below (figures 2-3). It is articulated around the Nios II processor. Several interfaces are used in order to connect the peripherals to processor (SPI, UART, Bus Avalon, PIO...).

The system is also composed of different sensors allowing the data acquisition, and the generation of the numerical signals. These signals are treated by target card ALTERA cyclone. After treatment, the control signals are routed towards a central station using a routing protocol.

Implementation routing protocol on MIS, can be done in two different ways, either directly into software, or hybrid: software part is C language and material acceleration implemented using hardware description language VHDL (optimizations to be made to answer the criterion of the consumption and the speed of execution). This implementation has been finalized and has been made possible by using in addition an operating system of the $\mu$ Clinux type. The big advantage of $\mu$ Clinux regard to its competitors is the compatibility of API's programming with the Linux standard systems. It also has all TCP/IP network functions, available on the Linux kernel and supported by the ALTERA card, Furthermore, it does not consume a lot of memory.

\section{QOS OF SERVICE MECHANISMS IN MIS NETWORK}

In the next subsections, we are going to describe mainly our contribution as the "Resource Optimization of Mobile Intelligent System with heart MPLS network", subject of this paper.

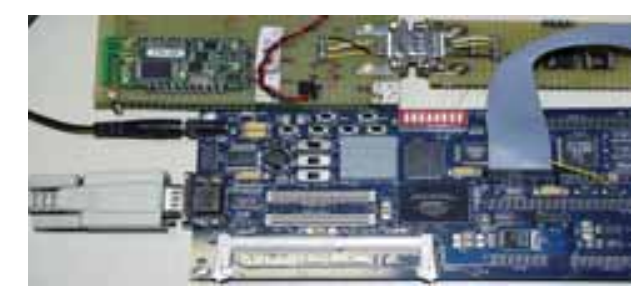

Figure 2. Wireless unit interfaced with the processing unit.

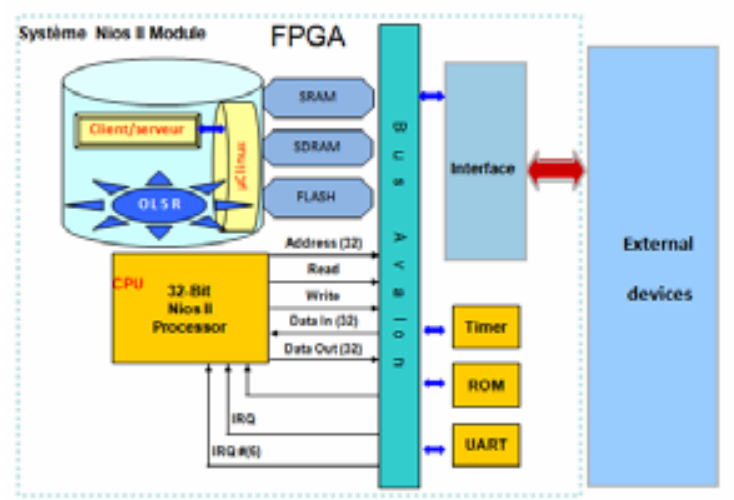

Figure 3. Synoptic of the System SoPC NIOS II for the acquisition and the routing of temperature

According to the recommendation E.800 CCITT, the quality of service is "the general effect of the performance of a service, which determines the degree of satisfaction of a user of the service" [1]. This definition is subjective and reflects the perception of the quality of service seen by a user. More technically, the quality of service is the ability to obtain a certain level of insurance so that traffic flow and/or services required are best satisfied, which allows you to share equitably and according to the needs required applications, all of the resources available in order to provide, wherever possible, each user quality he needs. Generally, this quality is focused on bandwidth, the delay and packet loss:

- In telephony networks, it is to be able to converse in real time (the time factor) without interruption caused by additional delays;

- In the Internet, to download a voluminous application does not ask more than to have a broad enough bandwidth to retrieve the file as soon as possible ;

Both applications are seeking (strongly or more flexibly) the integrity of all packets to the receipt.

For maximum reliability, QoS requires the cooperation of all segments in the active network, as well as each network element, from the beginning to the end. The different management policies are adopted to ensure QoS, depending where it is placed in OSI layers. To improve QoS in a MIS/MPLS/DiffServ networks, we need to address the IP layer:

\section{A. DiffServ Protocol}

Defined by the IETF, DiffServ (Differenciated Services) is a protocol designed to integrate the basic components of QoS management in IP networks. DiffServ acts at the level of traffic segmentation into several classes (classes of service). Resources allocation is done by class rather than by application [8]. Following the class to which a packet belongs, a more or less privileged treatment is applied to it (this includes priority routing, the 
selective removal of the packages in case of congestion). This solution is much less privileged than the approach of integration services (IntServ), which gives explicit reservation for each data flow. DiffServ sets three types of service classes [1]:

- EF (Expedited Forwarding): is the class of excellence. The EF packages must be sent with a guaranteed delay, jitter and a minimum rate of loss,

- AF (Assured Forwarding): AF four classes were defined, each of them has three subclasses. Packages are labeled AFxy where $\mathrm{x}$ in the interval [1.4] and $\mathrm{y}$ is the class number AF and in [1.3] precedence to spacing. Packets of the same class borrow the same queue to avoid inverse sequencing,

- BE (Best Effort): no special treatment.

In general, we count 14 possible behaviours, they are graded PHBs (Per Hop Behavior). A PHB is the way in which a router will treat incoming packets (ie put in queue more treatment in the event of congestion). The PHB is derived from DSPCs coded directly in the field of IP packet TOS.

\section{B. MPLS Protocol}

MPLS is a new technology, developed and standardized by the IETF, using switching mechanisms in order to reduce the cost of routing in the network layer while giving it better performance, greater scalability and greater flexibility in the restoration services [2] [9]. This technology is easily integrated with others such as DiffServ and InterServ.

The increased flow of information passing over networks, particularly the Internet, has spawned a need for bandwidth consumption growing in recent years. This demand has encouraged the evolution of these different networks which at the same time increased the complexity of managing them.

In terms of improvements, MPLS allows better management in routing (switching) and transfer packages, through networks [8]. But that's not because MPLS is able to solve many problems outlined above by improving four major aspects [8]:

- Possibility to define in advance the path that will take data or types of data sent over the network (Traffic Engineering).

- Ease of creating tunnels and IP VPNs (Virtual Private Network) level including Internet service providers, and solving problems related to the multiplication of them.

- Independence of layers 2 and 3 protocols with a support of IPv6, IPv4 layer 3, and Ethernet, Token Ring, FDDI, ATM, Frame Relay and PPP layer 2 .

- Interaction and cohabitation with existing routing protocols such as OSPF (Open Shortest Path First) and BGP (Border Gateway Protocol).

The MPLS architecture is based on switching labels mechanisms linking Layer 2 of the OSI model (switching) with the layer 3 of the OSI model (routing). Moreover, switching conducted layer 2 is independent of the technology used.

The networks now analyses IP headers to make routing decisions but MPLS based its decision on two distinct components: the control plan and data plane.
- The data plan can be used to transmit data packets based on labels, that are maintained in a database distributed on all intermediate routers.

- The control plan maintains information transmission labels to groups of switches labels.

Philips and all [10] limits the role of MPLS in the satisfaction of users requirements to differentiate and secure packets in the network.

Nagao and all [11] presents MPLS as a mechanism to administer routes by using optical networks, in order to achieve greater flexibility and change without interruption using secondary LSPs.

Haci and all [12] deals with the benefits of coupling DiffServ and MPLS to give several options for routing and increase the capacity of network resources to meet various needs of the users.

MPLS allows the network administrator to specify how to aggregate DiffServ (DiffServ Aggregates Behavior: BAs) with allocated LSPs. In fact, the question is how to allocate a set of BA to a set of LSPs. When packets arrive in the Ingress MPLS domain, the DSCP field is added to the header which corresponds to Aggregate Behavior (BA).

At each crossed node, the DSCP field will allow to select the right PHB (Per Hop Behavior), that defines the process scheduling (class of priority, WFQ.), and the probability of rejection. This mapping between MPLS classes and DiffServ PHB opens the door for interconnecting these different QoS mechanisms.

\section{Simulation}

These simulations presume to test some interconnection scenarios between MIS environments and IP QoS networks (DiffServ and MPLS) to anticipate problems that may arise in order to implement the best scenario suited to the users needs.

The aim of our simulation is to compare the use of MPLS or/and DiffServ protocols based on some QoS criteria such as: bandwidth, delay, loss and Signalling.

\section{A. Scenarios}

We valued the improvement of parameters of QoS of the MIS network, in order to analyze its compatibility with mechanisms of QoS (DiffServ, MPLS) inserting the effect of MIS mobility, while adopting the different applications; tcp connected mode with an acknowledgment that requires messages of signaling, in fact the loading of the network. However, cbr, ftp or udp no connected mode with minimum of signaling [14].

In our study, and to get a clear view and to extract relevant results, we will build our simulations on different traffic scenarios (figure 4):

- In the first, we took twelve MIS (risk area) transmitters linked to two AP (Access Point), generating a stream CBR / TCP and in the other side of the backbone, two mobile receptors connected by an AP;

- The second scenario includes twelve MIS transmitters linked to two AP, generating a stream CBR / UDP and the other side of the backbone two MIS receptors connected by an AP.

- In the third scenario, we took twelve MIS generate a stream FTP / TCP and the other side of backbone two MIS receptors, 
- The fourth scenario contains twenty MIS linked to both AP, half of which generate a stream CBR / TCP and the other FTP / TCP, and the other side of backbone two receiving MIS,

- The last case study is done on the same previous architecture with a change in access point with different speeds.

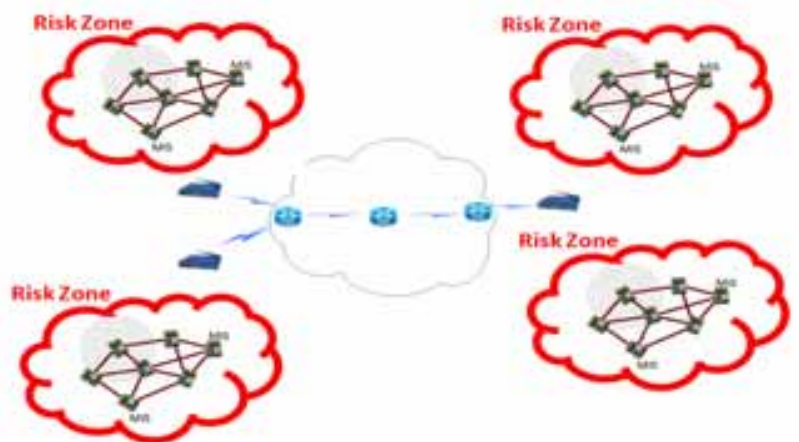

Figure 4. Simulation Architecture

These five scenarios were tested in different interconnection cases:

- WiFi/IP interconnection,

- WiFi/DiffServ interconnection,

- Wifi/MPLS interconnection,

- WiFi/Diffserv/MPLS interconnection.

\section{B. Simulation Results}

In this sub-section, we will present and discuss simulation results of the five scenarios discribed before. The scenario -1- will be presented in details; the other scenario will be presented in summary (because of the huge number of figures).

\section{Scenario 1:}

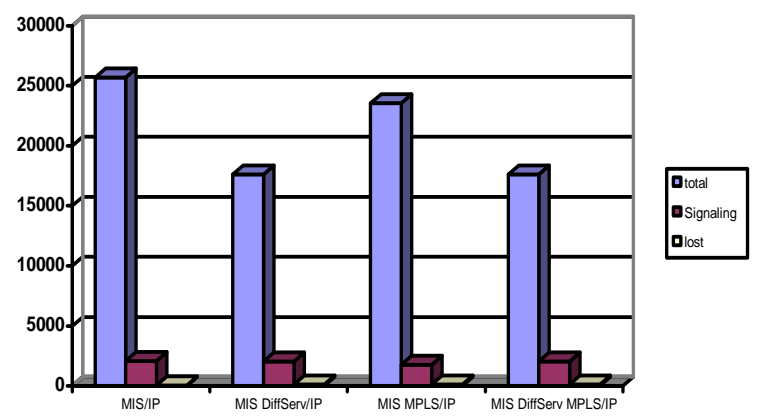

Figure 5. Packets load in QoS IP networks

Before comparing the results of the architecture, we will start our study based architecture with MIS / IP usual. In this architecture, the number of the total package is 25711, which Signal 2064 lost 47. on observing the figure (6) the flow reached $750 \mathrm{kbit} / \mathrm{s}$ in flow 11(sink 11) with delay very slow.

- MIS/DiffServ interconnection: there is a significant decrease of packets load, delay, throughput and signalling,

the number of the total package is 17619 , which Signal 2017 lost 126, with the fast delay in comparison with the case of MIS / IP with little change in bandwidth

- MIS/MPLS interconnection: there has been an increase in packets load accompanied with a decrease in delay, and a sharp decline in packets loss and signalling,

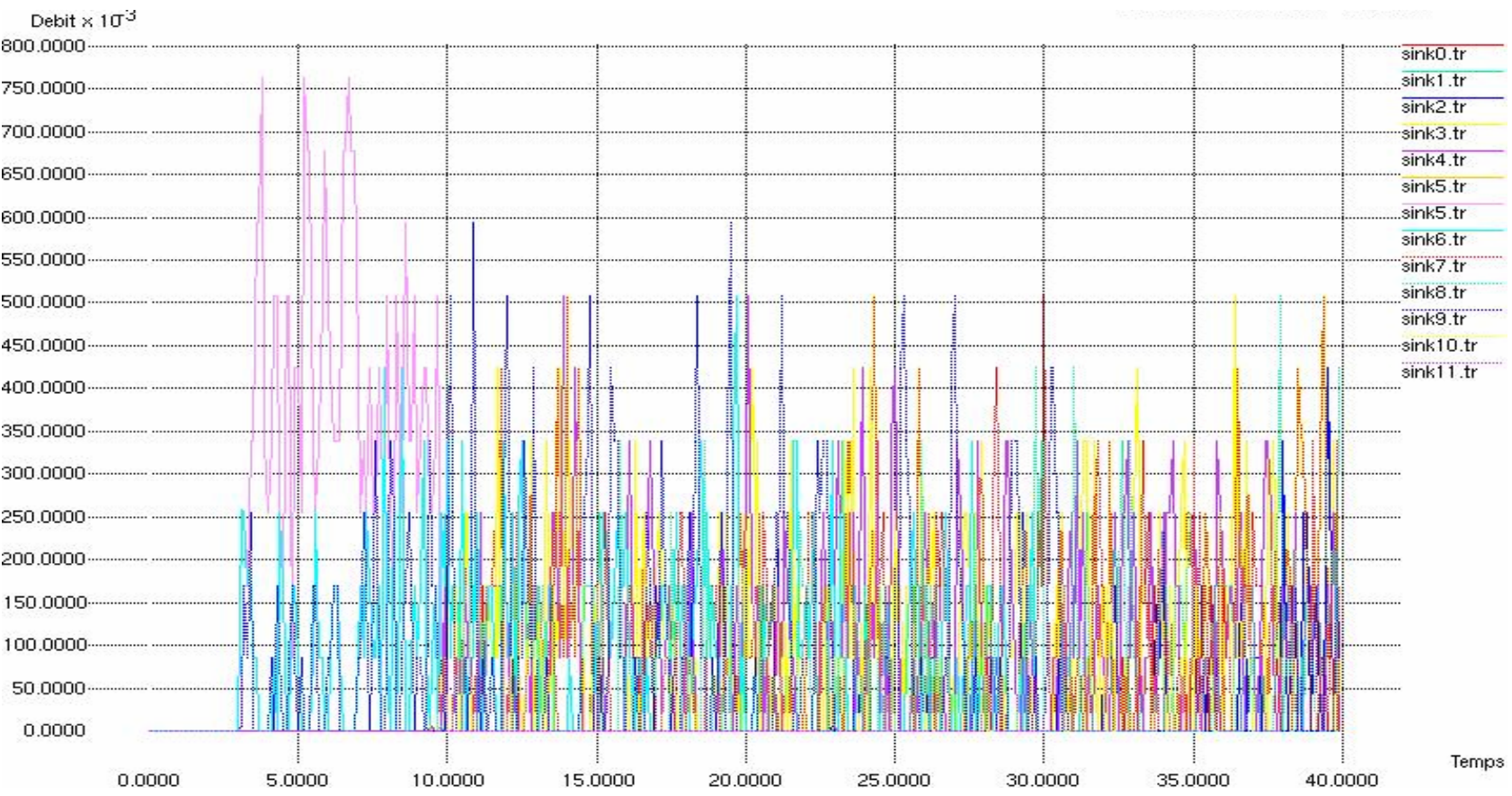

Figure 6. Bandwidth evolution over a MIS/IP network 


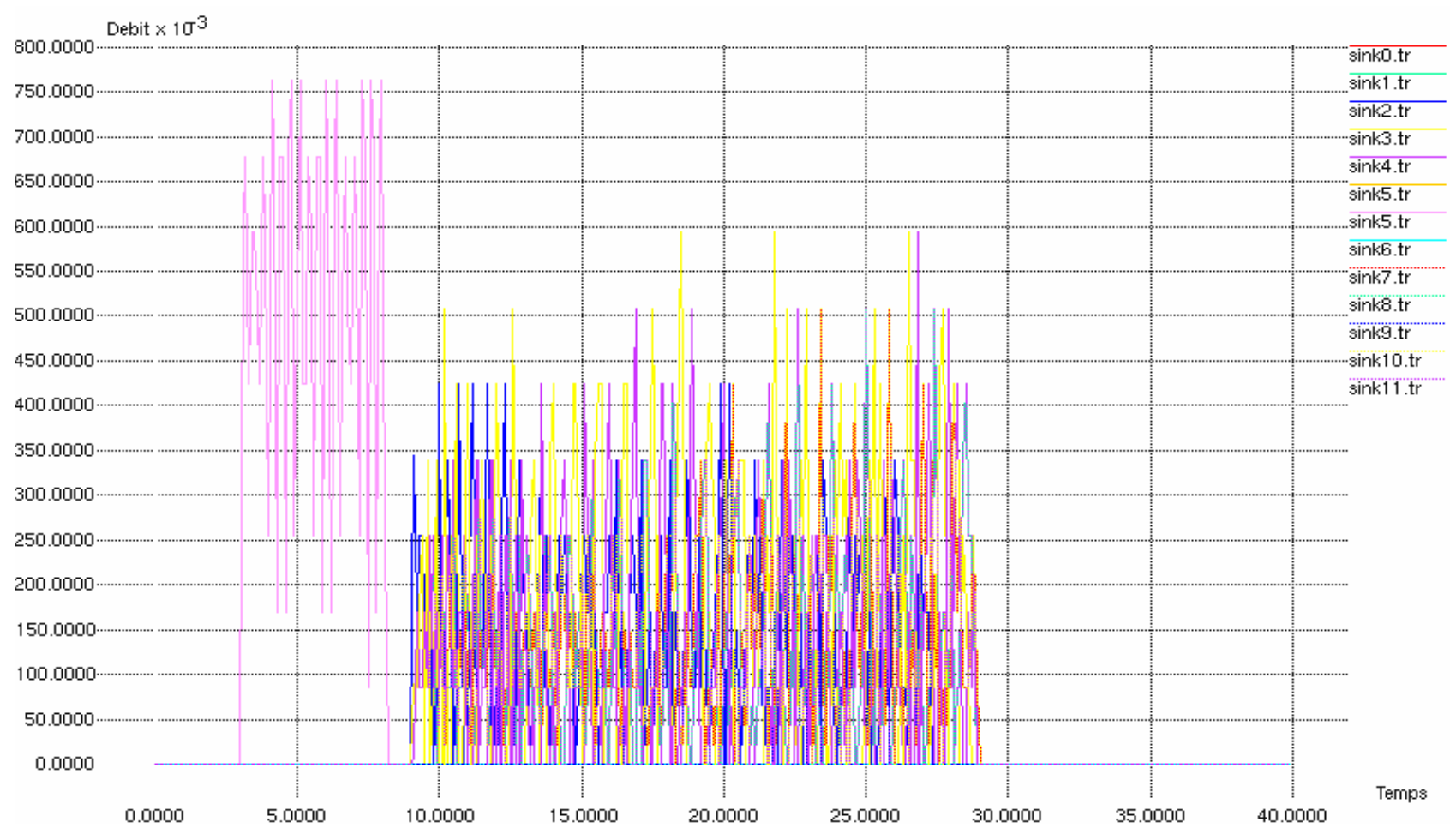

Figure 7. Bandwidth evolution over a MIS/DiffServ network

Debit

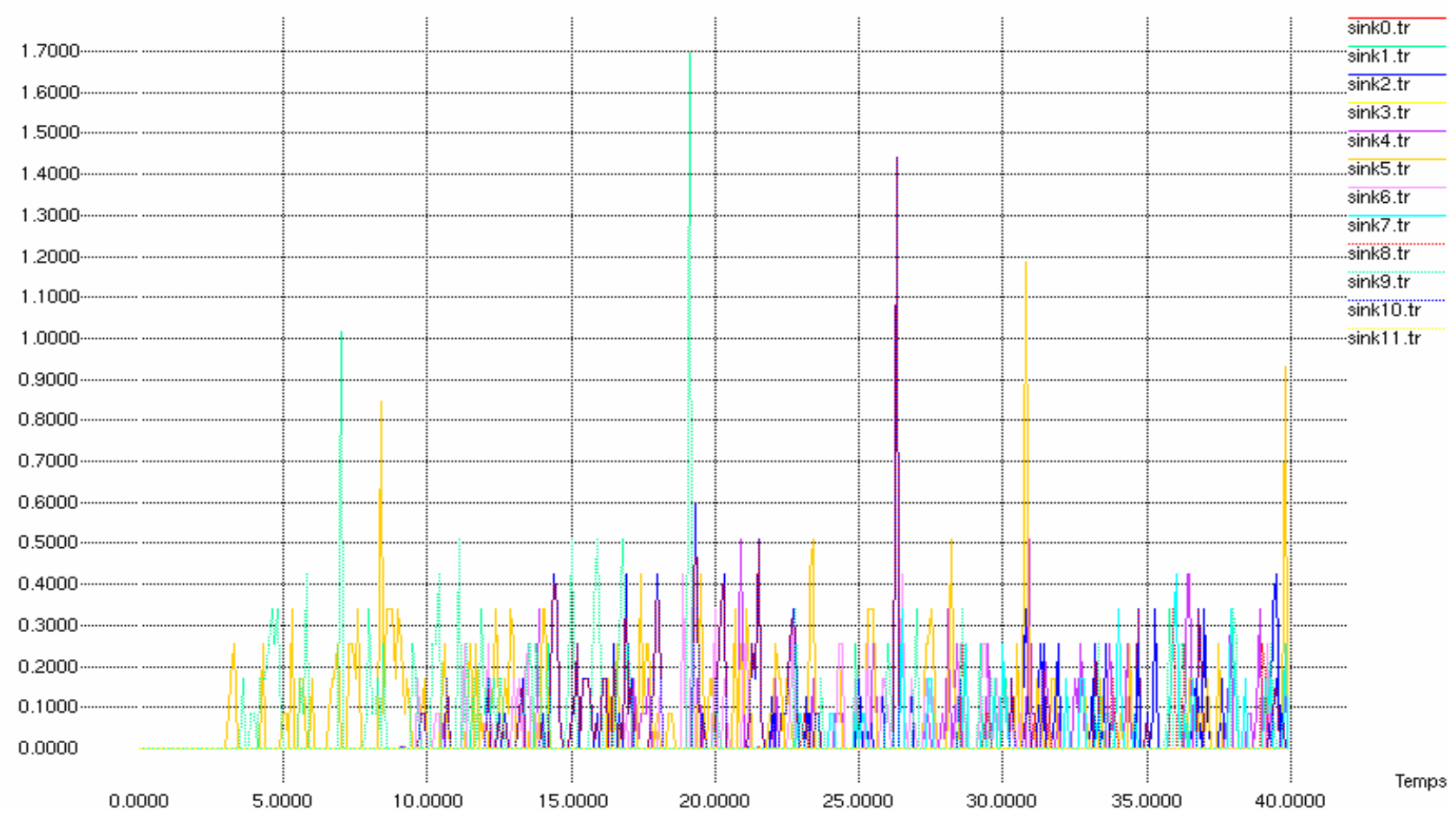

Figure 8. Bandwidth evolution over a MIS/MPLS network 


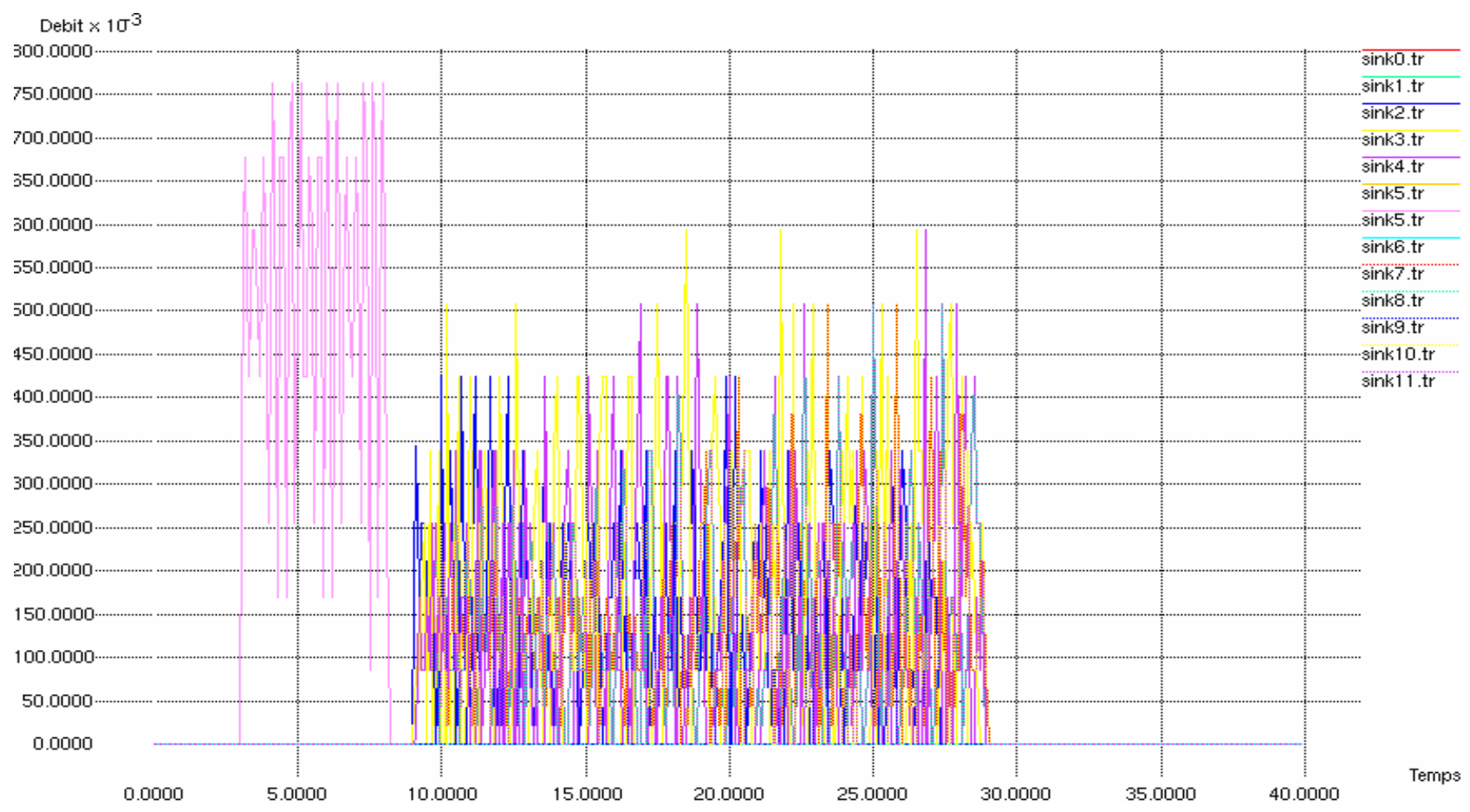

Figure 9. Evolution bandwidth as a function of time over a network MIS_Diff_MPLS

Increasing in Bandwidth (Figure 8) compared with MIS/IP with delay (174) very slow and also increasing in the number of packets because MPLS requires the message of signalisation for across the packets.

- MIS/DiffServ/MPLS interconnection: In this architecture the combination of MPLS and DiffServ gives the same statistics as the DiffServ mechanism operates only,

MPLS and DiffServ are not compatible

We have the same results in the case of MIS/DiffServ; DiffServ impedes the functioning of MPLS. you must use one to improve a given criterion (delay or bandwidth).

According to the obtained results in the MIS/IP architectures (figure: 5, 6, 7, 8, 9), we can conclude in different interconnection contexts:

In case of MPLS architecture, the increase in the throughput flow comes from the reduction of signalling and loss packets to begin the transmission of data (up). In the case of DiffServ, it is an improvement of QoS with a slight impact in comparison with the MPLS architecture because of the load of memory due to the data processing in relation to the DiffServ router and also the mode of transmission TCP (connected). On this, the coupling gives us the same result in DiffServ architecture.

\section{Scenario 2:}

- MIS/DiffServ interconnection: strong reduction packets load as well as in delay, with reduced number of packets lost and signalling;

- MIS/MPLS interconnection: increased number of packets, packet loss and delay, and the rate of signalling because of the process of building database of labels;
- MIS/DiffServ/MPLS interconnection: decrease in the throughput as a result of the operation of DiffServ, with increasing in flow with decreased delay.

Depending on these results, DiffServ architecture remains the best choice in case of CBR flows in a non-connected UDP, or rather without important signalling, is the environment preferred by the DiffServ.

Scenario 3:

- MIS/MPLS interconnection: strong reduction package as a whole as well as in the transmission rate, with the cancellation of lost packets and decreasing in packages signaling;

- DiffServ or coupling DiffServ/MPLS: gives us no effect.

In case of FTP / TCP remains the MPLS protocol single to improve QoS in compatibility mode with the TCP connection, which requires the security of transmission by using the signalling. This criterion meets with the data transfer protocol FTP criterion.

Scenario 4:

- MIS_MPLS interconnection: in this case, there is a decline in the number of packets whose lost packets and an increase in the bandwidth;

- DiffServ or DiffServ_MPLS interconnection: gives us no effect.

The combination of the two types of flows FTP / TCP and CBR / TCP provides the best environment for the operation of the connected mode (TCP) MPLS and bad result in the case of DiffServ, which supports our previous interpretation.

Scenario 5: 
- MIS/MPLS interconnection: in this case, there is a reduction in the number of packets and lost packets, and an increase in the throughput;

- DiffServ or DiffServ/MPLS interconnections: give us no effect.

The movement of stations has no effect on our scheme, because our work is the heart observation of the network operator, in which the question of compatibility arises naturally, without neglecting the effect of mobility whole.

Table 1 shows a summry of the obtained results when using different QoS architectures (DiffServ and MPLS)

TABLE I.

COMPARING SCENARIOS BASED ON QOS MECHANISMS.

\begin{tabular}{|l|l|l|}
\hline \multirow{2}{*}{ flow Type } & \multicolumn{2}{|c|}{ Mechanism QoS } \\
\cline { 2 - 3 } & \multicolumn{1}{|c|}{ DiffServ } & \multicolumn{1}{c|}{ MPLS } \\
\hline CBR/tcp & + & - \\
\hline CBR/udp & + & - \\
\hline FTP/tcp with & + & - \\
\hline $\begin{array}{l}\text { CBR \& FTP wandom movement } \\
\text { rand }\end{array}$ & + & - \\
\hline $\begin{array}{l}\text { CBR \& FTP with a } \\
\text { change in access point }\end{array}$ & + & \\
+ : increase in QoS \\
- : Decrease in QoS
\end{tabular}

\section{CONCLUSIONS}

In this paper, we have introduced the architecture of an intelligent beacon for ad-hoc wireless sensor networks named MIS (Mobile Intelligent System) and its first implementation on the platform. This beacon may acquire environmental data of the environment and detect possible defaults (great variations). When some alarm is triggered, data are sent on a wireless network such as Bluetooth Or Wifi.

In this work we have also to look for compatible mechanisms for QoS (DiffServ and MPLS) when using MIS to improve end-to-end performances. To achieve this goal, we have studied five different architectures (CBR, FTP, TCP, UDP, random movement or change access point);

According to the obtained results, we can conclude that the use of these protocols should be impacted by the application. We showed that network performances depends more on the application QoS parameters (flow, types of nodes, speed mobility, architecture and types of network layers used ...).

In the future works, we propose to study in detail the influence of these parameters on the performance of MIS, by trying to improve the performance of MPLS by using multi-path LSP routing.

The interest of such a work has a big impact for the applications related to the networks of wireless mobile sensors, in particular those dedicated to the military domain.

\section{REFERENCES}

[1] [RFC2475]: « Architecture for Differentiated Service», Dec 1998.

[2] Rosen E., Viswanathan A., and Callon, R. Multi-protocol Label Switching Architecture, IETF, RFC 3031, 2001.

[3] Habbani, A., Romain, O., El abbadi J And Garda, P. Circuit électronique de routage. Invention Brevet, OMPI 28185, 2005.

[4] Habbani A., Romain O., El abbadi J And Garda, P. Réseaux de capteurs Système d'acquisition et de routage d'information, international electronic revue in technologies of information (eTI). (Rabat), 2006.

[5] Habbani A., El abbadi J And Garda P, A versatile platform for AD-HOC, wireless sensor network node in FPGA, International Journal in Physics and Chemistry PCN (Physical and Chemical News). (EL JADIDA), 2007.

[6] Habbani, A., El Abbadi, J and Guennoun, Z. MIS: Mobile Intelligent System, IJCSNS (International Journal of Computer Science and Network Security), (Korea), 2007.

[7] Habbani A., Romain O And Garda P. MIS and I2C Bus, IJCSES (International Journal of Computer Sciences and Engineering Systems), (China), Vol.1, No.2, 2007.

[8] [RFC3270]: «MPLS-Support of Differentiated Services».

[9] [RFC3032]:
http://www.ietf.org/rfc/rfc3032.txt?number=3032.

[10] Phillips C., Bigham, J. He L. and Littlefair B., Managing dynamic automatic communities with MPLS-based VPNs, BT Technology Journal, Vol 24 No 2, April 2006. (doi:10.1007/s10550-006-00429)

[11] Ogino, $\mathrm{N}$ and Tanaka, H. Optimum arrangement of reliable labelswitched paths in MPLS over optical networks, Springer Science+Business Media, pp: 29-41, 2006.

[12] Haci, A. and Mantar, A., scalable QoS routing model for diffserv over MPLS networks, 7 June 2007, Springer Science and Business Media, pp: 107-116, 2007.

[13] Haci A. and Mantar A, scalable QoS routing model for diffserv over MPLS networks, Published online: 7 June 2007 Springer Science+Business Media, pp: 107-116, 2007.

[14] Oubaha J. and Elkoutbi M, 802.11 Mobile Networks Combined to QoS IP Networks, ICTTA Information and Communication Technologies: From Theory to Applications, IEEE Society, pp: 1-5, April 2008, Damascus.

\section{AuTHORS}

Jawad OUBAHA is with SI2M Laboratory, E.N.S.I.A.S, Mohammed V-Souissi University B.P. 713 Rabat, Morocco.

Ahmed HABBANI is with SI2M Laboratory, E.N.S.I.A.S, Mohammed V-Souissi University B.P. 713 Rabat, Morocco and Laboratory LEC, Ecole Mohammadia d’Ingénieurs Avenue Ibn Sina B.P. 765 Agdal Rabat - Morocco.

Mohammed Elkoutbi is with SI2M Laboratory, E.N.S.I.A.S, Mohammed V-Souissi University B.P. 713 Rabat, Morocco.

Submitted 10 June 2009. Published as resubmitted by the authors on 9 October 2009. 